\title{
Heat transfer behind the backward-facing step under the influence of longitudinal pressure gradient
}

\author{
Tatyana Bogatko*, Viktor Terekhov, Aleksey Dyachenko, and Yaroslav Smulsky \\ Kutateladze Institute of Thermophysics SB RAS, 630090, Novosibirsk, Russia
}

\begin{abstract}
Results of an experimental and numerical study of the flow structure and turbulent heat transfer in a rectangular channel with longitudinal pressure gradient behind the backward-facing step. With the channel expansion the angle of rotation of the upper wall is $1.43 ; 2.86$ and $4^{\circ}$, and with the channel convergence the rotation angle is $3 ; 5.7$ and $7.6^{\circ}$. Experiments are carried out at Reynolds numbers, calculated on the step height and velocity in front of the backward-facing step, $\mathrm{Re}_{\mathrm{H}}=4000 ; 8$ $000 ; 12000$. It was determined, with the increase of pressure gradient the maximum value of Nusselt number increases for the converging channel and decreases for the expanding channel.
\end{abstract}

\section{Introduction}

This study focuses on the properties of a separated flow behind the backward-facing step under conditions of imposed longitudinal pressure gradient. The most common are channels with pressure gradient in the flow paths of compressors. These are interblade channels of impellers, guide vanes and straighteners, as well as transition channels with different configuration of the flow part. Separation usually leads to harmful consequences, causing loss of energy, onset of instability, etc. To further improve the efficiency of thermal power equipment and to develop the fundamentals of separated flows it is crucial to conduct comprehensive experimental and computational studies of new opportunities of intensification of heat and mass transfer in the separation gradient flows behind the backward-facing step. Of interest are both expanding and converging channels. This report contains the results of an experimental study of the separated flow behind the backwardfacing step in a flat channel with flow acceleration and deceleration. The longitudinal gradient was created due to the position of the upper channel wall behind the backwardfacing step. The pressure fields and heat transfer coefficients in recirculating and relaxation areas behind a drastic channel expansion were measured. It should be noted that data on similar themes available in the literature concern the flow dynamics [1-3], and data on heat transfer are virtually absent.

The report also presents results of the numerical simulation of the effect of positive and negative longitudinal pressure gradients on hydrodynamic flow characteristics and turbulent

${ }^{*}$ Corresponding author: tbogatko@gmail.com 
heat transfer in a pipe with sudden expansion. A theoretical approach to the description of turbulent separated flows is rather complicated and remains topical to the present time. Extensive research for creating the engineering methods of separated flow calculation was conducted in [4-6]. The calculations were performed within the model of incompressible fluid based on the system of Reynolds averaged stationary equations of Navier-Stokes and energy equations.
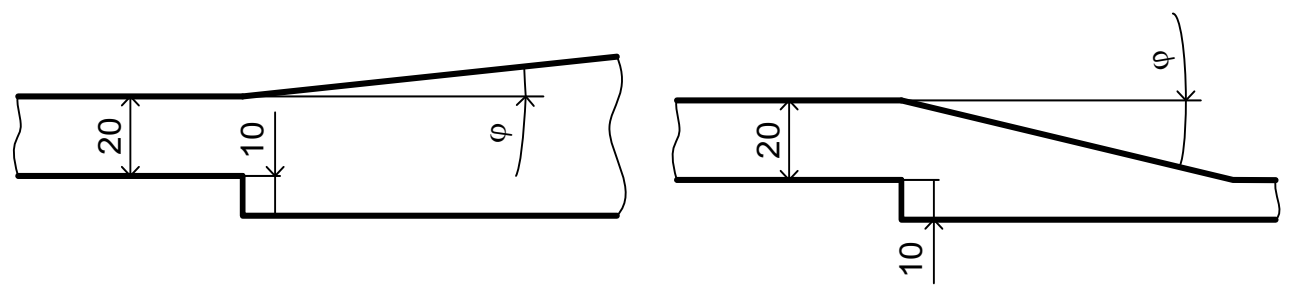

Fig. 1. Schematic diagrams of experimental section in an expanding (left) and converging (right) channels.

Fig. 1 shows schemes of experimental measurements in expanding and converging channels. With the channel expansion the angle of rotation of the upper wall is $1.43 ; 2.86$ and $4^{\circ}$, and with the channel convergence the rotation angle is $3 ; 5.7$ and $7.6^{\circ}$. In the latter case, the length of the inclined wall $(\mathrm{L}=380 ; 200$ and $150 \mathrm{~mm})$ decreases, and its lower end is always located at a distance of $10 \mathrm{~mm}$ from the bottom channel wall. Experiments are carried out at Reynolds numbers, calculated on the step height and velocity in front of the backward-facing step, $\mathrm{Re}_{\mathrm{H}}=4000 ; 8000 ; 12000$. The calculations required for the work are performed using the k- $\omega$ SST turbulence model [4], most adequately describing the turbulent separation flows [5]. The problem statement is two-dimensional, and the flow is stationary. The geometry and initial conditions correspond to experimental parameters.

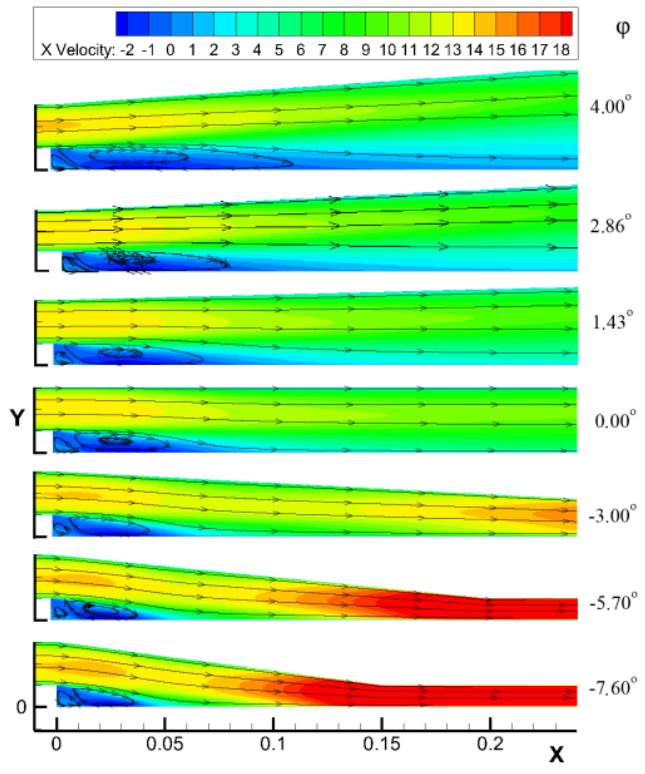

Fig. 2. Velocity field in expanding and converging channels, $\mathrm{Reh}=8000$.
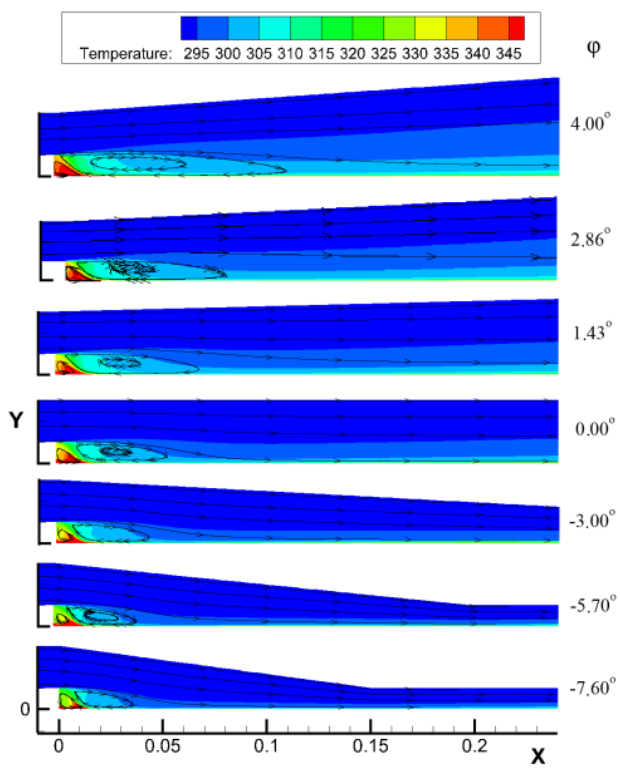

Fig. 3. Temperature field in expanding and converging channels, Reh $=8000$. 


\section{Results}

For different values of the longitudinal pressure gradient, the flow character behind the backward-facing step varies considerably. The main difference is observed along the length of the recirculation zone. Fig. 2 shows the flow patterns in the region of sudden expansion and the field of the longitudinal velocity component. It is seen that the length of the recirculation zone increases with increasing adverse pressure gradient and greatly reduces at imposed favorable pressure gradient. In addition, this figure clearly shows the deceleration and acceleration of the flow at a turn of the upper cover of the channel. In this section of the channel convergence there is a significant, two-fold acceleration of the flow.

Fig. 3 with streamlines superimposed on the temperature field clearly demonstrates how the thermal boundary layer increases (at decelerating flow) and decreases (at accelerating flow). Accordingly, the heat removal from the surface in this section of the channel varies significantly.
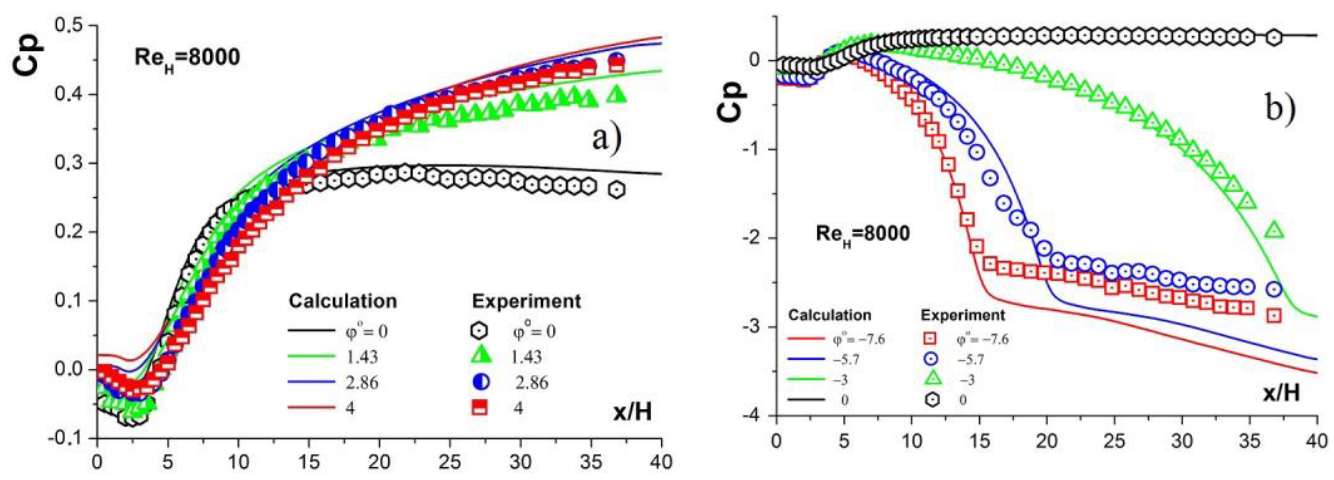

Fig. 4. Distribution of the pressure coefficient in the expanding (a) and converging (b) channels.

Distributions of pressure coefficients for both cases are shown in Fig. 4. With an increase in the angle of channel expansion the pressure ratio increases, but the increase takes place almost to the value $\varphi=2.86^{\circ}$. A further increase in the angle has no influence on the value of pressure coefficients. With the growth of the channel convergence angle the pressure ratios decrease. When $\mathrm{L}=200$ and $150 \mathrm{~mm}\left(\varphi=5.67\right.$ and $\left.7.5^{\circ}\right)$ the pressure distribution along the confusor length changes, and the region of flow relaminarization is clearly seen.
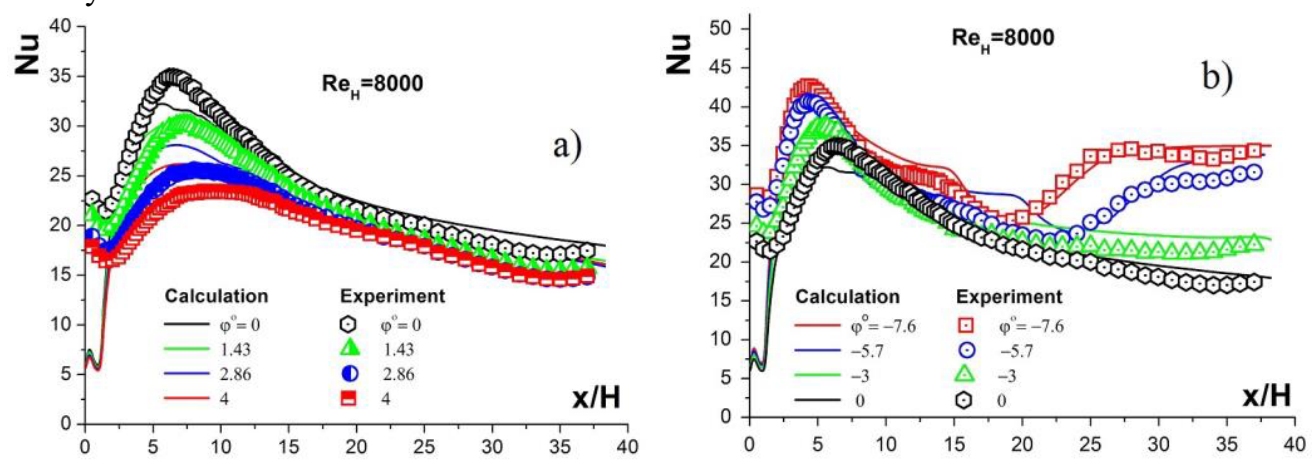

Fig. 5. Distribution of heat transfer coefficient in the expanding (a) and converging (b) channels.

Fig. 5 demonstrates changes in the Nusselt number along the longitudinal coordinate behind the backward-facing step. At adverse pressure gradient, with increasing angle the flow decelerates increasingly, and the maximum heat transfer coefficient decreases. 
At favorable pressure gradient the situation is opposite. Maximum heat transfer increases, but less intensively than in case of adverse pressure gradient. At $x / H=25-30$ for angles $\varphi=5.67$ and $7.5^{\circ}$, similar to pressure ratios, the Nusselt numbers clearly reflect the pronounced flow relaminarization.

Figure 6 shows maximum heat transfer coefficients depending on the Kays parameter $K=\frac{v}{U_{0}^{2}} \frac{d U_{0}}{d x}$.

flow, negative values correspond to diffuser flow, and positive values correspond to confluence.
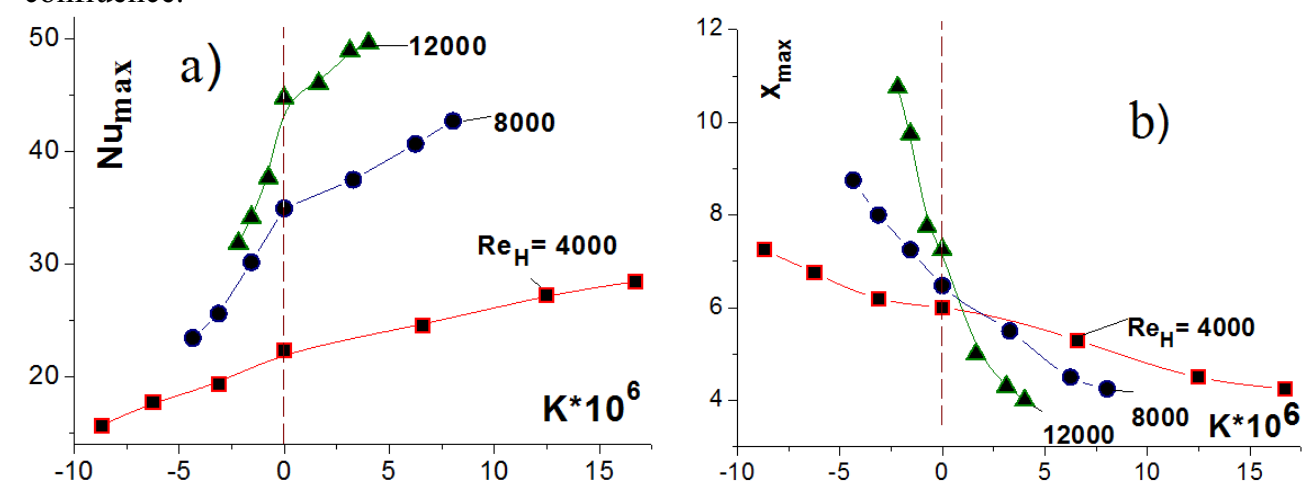

Fig. 6. Dependence of maximum heat transfer coefficient (a) and its coordinate (b) in the expanding and converging channels on Kays parameter.

With the increase of pressure gradient the maximum value of Nusselt number increases for the converging channel and decreases for the expanding channel. The dependence of the Nusselt number on the Reynolds number is apparent. At increased Reynolds number, not only the absolute value of $\mathrm{Nu}_{\max }$, but the angle of the lines describing the experimental data increase. This suggests that with increasing Reynolds number the effect of longitudinal pressure gradient on heat transfer is greater.

An important parameter in the separation flow is the length of the return flow area, which correlates with the length of the maximum heat transfer $\mathrm{x}_{\max }$. In accordance with Fig. 6 , the value of $x_{\max }$ increases at growing adverse pressure gradient and decreases at an increase in favorable pressure gradient. In this case $\mathrm{x}_{\max }$ is strongly dependent on the Reynolds number.

As can be seen from Fig. $6 \mathrm{~b}$, at zero-pressure-gradient flow $(K=0)$ the Re number influences the position of the maximum heat transfer $\left(\mathrm{x}_{\max }=6-7.5\right)$, which is confirmed by experiments of other authors [6-8].

In accordance with Fig. 6b, the gradient flows have qualitatively different flow patterns with a strong dependence on the $\mathrm{Re}_{\mathrm{H}}$ number.

This work was supported by the Russian Science Foundation (Project RNF 14-19-00402).

\section{References}

1. Lo, Kin Pong, J.E. Christopher, K.E. John, Exp. fluids 53, 1317 (2012)

2. D.M. Driver, H.L. Seegmiller, AIAA J. 23,163 (1985)

3. D.A.D. Kuehn, AIAA J. 18, 343 (1980)

4. F.R. Metnter, AIAA J. 32, 1598 (1994) 
5. V.I. Terekhov, T.V. Bogatko, J. Appl. Mec. Tec. 56, 471 (2015)

6. A.I. Leontiev, S.I. Kaskov, Yu.F. Gortyshov, I.A.Popov, V.V. Olimpiev, ASME, Heat Transfer Division (2006)

7. V.K. Migay, Modelling of heat exchange power equipment (Energoatomizdat, Moscow, 1987)

8. Yu.F. Gortyshov, I.A. Popov I.A., Therm. Eng. 53, 249 (2006) 